\title{
Electrochemical Sensing Platform for Glutamic Pyruvic Transaminase Determination
}

\author{
Fangfang Yao ${ }^{1,2}$, Pengyuan Zheng ${ }^{1, *}$, Huang Huang ${ }^{1}$, Yong $\mathrm{Yu}^{1}$, Lu Mei ${ }^{1}$ and Runping $\mathrm{Jia}^{2}$ \\ ${ }^{1}$ The Fifth Affiliated Hospital of Zhengzhou University, Zhengzhou, Henan, 450052, P.R. China \\ ${ }^{2}$ The Second Affiliated Hospital of Zhengzhou University, Zhengzhou, Henan, 450014, P.R. China \\ *E-mail: zhengypfifth@ sina.com
}

doi: $10.20964 / 2018.03 .28$

Received: 4 November 2017 / Accepted: 17 December 2017 / Published: 5 February 2018

\begin{abstract}
The activity of glutamic pyruvic transaminase (GPT) is a clinically significant factor for some acute diseases (e.g., acute hepatopathy and myocardial infarction), necessitating the fast and small format analysis of GPT at point-of-care. In the present study, a graphene-Nafion nanohybrid film coated sensor was used to detect GPT. A simple drop-casting method was used for the preparation of Nafion graphene oxide-decorated screen-printed electrode (SPE), while an electrochemical strategy was employed to reduce the graphene oxide on the SPE surface. Our proposed sensor can determine GPT over the range of 5-300 U/L, with the limit of detection (LOD) of $2.2 \mathrm{U} / \mathrm{L}$.
\end{abstract}

Keywords: Electrochemical sensing; Glutamic pyruvic transaminase; Graphene; Liver disease; Clinic diagnosis

\section{$\underline{\text { FULL TEXT }}$}

(C) 2018 The Authors. Published by ESG (www.electrochemsci.org). This article is an open access article distributed under the terms and conditions of the Creative Commons Attribution license (http://creativecommons.org/licenses/by/4.0/). 